\title{
Reproducibility of corneal astigmatism measurements with a hand held keratometer in preschool children
}

\author{
Erin M Harvey, Joseph M Miller, Velma Douson
}

\begin{abstract}
Aims-To evaluate the overall accuracy and reproducibility of the Alcon portable autokeratometer (PAK) measurements in infants and young children.

Methods-The accuracy of the Alcon PAK in measuring toric reference surfaces (1, 3,5 , and 7 D) under various suboptimal measurement conditions was assessed, and the reproducibility of PAK measurements of corneal astigmatism in newborn infants $(n=5)$, children $(n=19$, age $3-5$ years), and adults $(n=14)$ was evaluated. Results-Measurements of toric reference surfaces indicated (a) no significant effect of distance (17-30 $\mathrm{mm}$ ) on accuracy of measurements, (b) no systematic relation between amount of toricity and accuracy of measurements, (c) no systematic relation between angle of measurement and accuracy, (d) no difference in accuracy of measurements when the PAK is hand held in comparison with when it is mounted, (e) no difference in accuracy of measurements when axis of toricity is oriented obliquely than when it is oriented horizontally, with respect to the PAK, and (f) a small positive bias $(+0.16 \mathrm{D})$ in measurement of spherical equivalent. The PAK did not prove useful for screening newborns. However, measurements were successfully obtained from $18 / 19$ children and $14 / 14$ adults. There was no significant difference in median measurement deviation (deviation of a subject's five measurements from his/her mean) between children $(0 \cdot 21 \mathrm{D})$ and adults $(0 \cdot 13 \mathrm{D})$.

Conclusions-The PAK produces accurate measurements of surface curvature under a variety of suboptimal conditions. Variability of PAK measurements in preschool children is small enough to suggest that it would be useful for screening for corneal astigmatism in young children.

(Br f Ophthalmol 1995; 79: 983-990)
\end{abstract}

Department of Optical Sciences

J M Miller

Department of Psychology V Dobson

Correspondence to: Erin Harvey, MA, University of Arizona College of Medicine, Department of Ophthalmology, $1801 \mathrm{~N}$ Campbell Ave, Tucson, AZ 85719, USA.

Accepted for publication 15 June 1995

Previous research has indicated that there is high prevalence of astigmatism greater than 1 dioptre (D) in infants, ${ }^{1-4}$ and that this astigmatism is due primarily to corneal, rather than lenticular, asphericity. ${ }^{5}$ Although the prevalence of astigmatism decreases greatly by the preschool years, ${ }^{4-7}$ it can, in some cases, lead to meridional amblyopia (MA), ${ }^{8}$ a deficit in acuity for stimuli of certain orientations that is present even when the patient is tested with optical correction. ${ }^{910}$

Although only a small number of individuals have been studied, there is evidence that MA can be prevented if astigmatic children receive optical correction before reaching school age. Mitchell et al, in a study of adult astigmats, found MA in all subjects who had received optical correction after 6 years of age, but not in the one individual who had received glasses at age $3 .{ }^{10}$ Similarly, Cobb and MacDonald, in a study of 12 astigmatic adults, found that those corrected after age 7 had MA while the majority of those corrected before age 7 did not. ${ }^{11}$ One child, tested by Mohindra et al, showed MA when first placed in glasses at age $\mathbf{3 4}$ months, but showed no evidence of meridional acuity deficits when tested after 3 months of wearing glasses. ${ }^{12}$ Thus, these results suggest that it may be valuable to identify and correct children for astigmatism before school age, to prevent the development of MA.

The traditional method for detecting astigmatism in preschool age children involves cycloplegic or non-cycloplegic retinoscopy. Recently, there has been an emphasis on the development of screening tools that can be used by lay individuals. One such tool is the portable autokeratometer (PAK) (Alcon Systems Inc, St Louis, MO, USA), a hand held instrument that measures corneal astigmatism. To date, there are no reports of the efficacy, validity, or reliability of the PAK for the measurement of the curvature of known toric reference surfaces or for the assessment of corneal astigmatism in children. However, Palmer and Reid examined reliability, validity, and operator bias of the PAK in a study of 12 adult patients. ${ }^{13}$ Palmer and Reid reported a $95 \%$ confidence interval of $0.48 \mathrm{D}$ for repeatability of meridional power measurements on individual patients, and validity coefficients of 0.96 for $\mathrm{K} 1$ and 0.99 for $\mathrm{K} 2$ in comparisons of measurements taken with the PAK versus those taken with the TMS-1 (Topographic Modeling System, Computed Anatomy, New York, NY, USA). There was no evidence of operator bias.

One aim of this study was to evaluate the presumed to be neural in origin, since it is overall accuracy of the PAK. In three experiments, we tested the accuracy of the PAK in measuring known toric reference surfaces under various measurement conditions. These experiments were designed to simulate and evaluate the influence of measurement conditions that might occur when obtaining measurements from young children who may be less than optimally cooperative patients. 
In the first experiment, we attempted to establish optimum working distance and to determine the sensitivity of the PAK to variation in working distance by varying the measurement distance from the toric surface. In the second experiment, we evaluated the influence of operator alignment error on the accuracy of the PAK by comparing accuracy of measurements taken with the PAK mounted in a fixed position to measurements taken with the PAK hand held. In the third experiment, we evaluated the accuracy of the PAK in a condition that simulated a subject not fixating on the PAK display. In addition, in experiment three, we compared accuracy of the PAK in measuring toric surfaces when the axis of toricity was oriented horizontally versus obliquely with respect to the PAK. Since the PAK uses horizontal and vertical measurements to calculate toricity, it was hypothesised that the PAK may be more accurate in measuring surfaces with horizontal and vertical axes of toricity than surfaces with oblique axes of toricity.

In the final portion of this study, we examined the reproducibility and ease of use of the PAK in the measurement of corneal astigmatism in infants and young children, in comparison with adult subjects.

\section{Methods}

\section{MATERIALS}

\section{Portable autokeratometer (PAK)}

The Alcon PAK is a hand held instrument for measuring corneal astigmatism. The instrument is designed for easy use by individuals with minimal ophthalmic experience.

The PAK employs the same optical principles as a single ring keratoscope. In a ring keratoscope, a ring of known diameter is placed at a known distance from the cornea. The radius of curvature of the cornea is determined by measurement of the magnification of the image of the ring reflected from the air/tear interface of the cornea. While the PAK does not use a continuous ring, it has four light sources that fall on a circular path. In the presence of a spherical cornea, the reflected images of the four lights also fall on a circular path. In the presence of corneal astigmatism, the reflected images of the four lights fall on an elliptical path. The computer within the PAK determines the orientation and size of the major and minor axes of the ellipse and uses the relative magnification produced by the cornea along these axes to determine corneal power.

The PAK provides measurements of the following aspects of corneal topography: base curve and axis of the most horizontal meridian (K1), base curve and axis of the most vertical meridian (K2), delta $\mathrm{K}$ (the amount of astigmatism), and the angle of delta K. During use, the PAK takes repeated readings of corneal topography. The instrument compares each set of five sequential readings as follows: it ignores the highest and the lowest of the five readings and, if the middle three readings agree to within $1 / 8$ dioptre, it terminates measurements and selects as its estimate of each aspect of corneal topography $(\mathrm{K} 1, \mathrm{~K} 2$, delta $\mathrm{K}$, axis of delta $\mathrm{K}$ ) the values obtained from the median of the five readings. Data from one eye are stored by the PAK until measurements of the patient's other eye have been completed. Results for both eyes are printed by placing the PAK in its charger/stand.

For patients who are sitting upright, the axis of astigmatism is referenced to vertical by a gravity sensor. For supine patients, the axis of the instrument handle serves as the reference for astigmatism axis measurements. The instrument was used in accordance with manufacturer's guidelines. ${ }^{14}$

\section{Toric reference surfaces}

The toric surfaces used in the evaluation of the accuracy of the PAK were four surfaces fabricated from Cleartran (CVS Advances Materials, Woburn, MA, USA), a water-free form of zinc sulphide, by single point diamond turning. Cleartran has a refractive index of approximately 2 , resulting in a Fresnel reflectivity in air of approximately $11 \%$. While Cleartran is significantly more reflective than the air/tear interface, which has a reflectivity of about $2 \%$, it is much less reflective than a steel ball bearing, the other frequently used calibration surface. It was not necessary to introduce neutral density filters into the optical system or to reduce the PAK sensitivity, in order to obtain measurements.

The reference surfaces were fabricated to have a base curvature of $43 \mathrm{D}$ and toricity equal to $1,3,5$, and $7 \mathrm{D}$. The curvatures were verified both by stylus profilometry and interferometry. ${ }^{15}$ Although the entire extent of the human cornea is not toric, we chose to use a toric reference surface for these measurements because the 'optical zone' of the cornea, overlying the natural pupil, may be modelled as a toric surface. The area of the corneal surface measured by the PAK is approximately that overlying a natural $(4 \mathrm{~mm})$ pupil.

\section{MEASUREMENTS OF TORIC REFERENCE SURFACES}

\section{Procedures}

All measurements of the toric surfaces were made by one examiner (JM). The toric reference surfaces were secured to a surface that contained a horizontal ruled line that served as a guide for aligning the axis of toricity. Frame mounted measurements were obtained by placing the PAK vertically in an optical bench stand. In order to obtain a printout, it was necessary to remove the instrument from the stand after every two readings. The examiner performed hand held measurements by aligning the PAK with the horizontal ruled line, thus approximating the task of repeated alignment of the same eye. In mounted measurements, the PAK and reference surface were oriented vertically (as if taking measurements from a patient sitting upright) and in hand held 
(top)

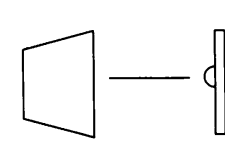

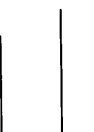

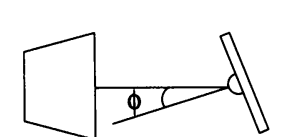

$\left[\begin{array}{l}P \\ A \\ K\end{array}\right]$

A

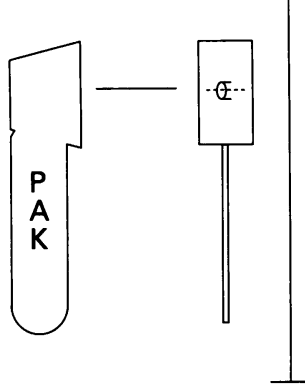

B
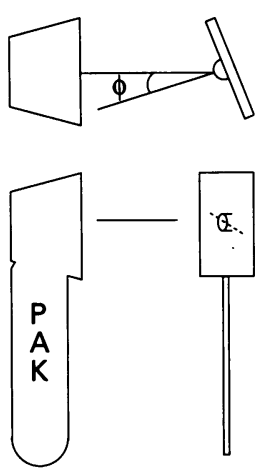

C

Figure 1 Illustration of experiment 3. (A) The toric reference surface is oriented towards the fixation target of the PAK. Angle $\theta$, the angle between the toric surface and the optical axis of the $P A K$, is 0 degrees, simulating a subject looking at the fixation target. (B) The reference surface is rotated away from the fixation target at angle $\theta(5,10,15$, or 20 degrees), simulating a subject looking to the left of the PAK. Axis of astigmatism is horizontal with respect to the $P A K$. (C) Reference surface rotated at angle $\theta$; astigmatism axis oblique with respect to the $P A K$.

measurements the PAK and reference surface were oriented horizontally (as if taking measurements from a supine patient). Gravity (determined by PAK gravity sensors) served as the reference for mounted measurements of axis of toricity; the handle of the PAK served as the reference for hand held measurements of axis of toricity.

In order to evaluate the effects of various measurement conditions on accuracy of the PAK, three experiments were conducted. In the first experiment, the PAK was mounted on a micrometer and the distance from the $5 \mathrm{D}$ toric surface to the face plate of the PAK was incrementally increased in $1 \mathrm{~mm}$ steps. One measurement was attempted at each distance. In the second experiment, 10 measurements of each the four toric surfaces were taken with the PAK fixed and mounted, and 10 measurements of each surface were taken with the PAK hand held. In the third experiment, the effect of a subject not looking precisely at the fixation target was simulated by rotating the $5 \mathrm{D}$ reference surface by $0,5,10,15$, and 20 degrees away from the PAK (see Fig 1). Measurements of the $5 \mathrm{D}$ reference surface were taken at the optimum working distance (determined in experiment 1). Two measurements were taken at each angle of eccentricity with the axis of toricity oriented horizontally with respect to the PAK (Fig 1B), and two measurements were taken at each angle of eccentricity with the axis of toricity oriented obliquely at 45 degrees with respect to the PAK (Fig 1C).

MEASUREMENTS FROM INFANT, PRESCHOOL, AND ADULT SUBJECTS

\section{Subjects}

Newborn subjects were five infants less than 24 hours of age, born at Tucson Medical Center in November 1994. Preschool subjects were 19 consecutive patients and siblings between 3 and 5 years of age seen at the University of Arizona Pediatric Ophthalmology Clinic or the Newborn Intensive Care Program

Developmental Follow up Clinic. Mean age of preschool subjects was 56 months, with a range from 38 to 71 months. Adult subjects were 14 volunteers ranging in age from 21 to 50 years (mean 35 years). Written informed consent was obtained from adult volunteers and parents of newborns and children.

\section{Procedure}

A single examiner (EH) attempted to obtain five consecutive measurements from one eye of each subject. Because we wanted to evaluate the instrument for screening, we chose an examiner who had minimal ophthalmological training. Before attempting measurements on experimental subjects, the examiner had limited experience in using the PAK with adults, and no experience using the PAK with newborns or preschool children. During testing of newborns, another examiner (VD) gently held the subject's eyelids open. Children and adults were asked to remain still and to fixate the centre red light in the display. Once fixation was achieved and the instrument was aligned, the examiner attempted a measurement. Data were printed after the second, fourth, and fifth measurements.

\section{Data analyses}

Analysis of the reproducibility and accuracy of astigmatism data is complicated by the interaction of sphere, cylinder, and axis measurements. Small axis variations of large cylinder powers can produce the same dioptric blur as are produced by large axis variations of small cylinder power. That is, variation of either sphere or cylinder power can produce variation in the net dioptric power.

Analysis of the variation of astigmatic power is best accomplished with the vectoral method described by Harris. ${ }^{16}$ Using this method, each observation of sphere, cylinder, and axis is converted into a single point in dioptric three dimensional space. The advantage of this method is that the deviation for each measurement can be derived in units of dioptres that are related simultaneously to the sphere, cylinder, and axis of the measurements.

Repeated observations result in a cluster of points occurring in this three dimensional space. For measurements of reference surfaces, dioptric distances of the observed points in the cluster from the point representing the actual dioptric power were used to determine measurement accuracy. Spearman correlation, Kruskal-Wallis ANOVA on ranks, and the Mann-Whitney rank sum test were used to evaluate differences in deviation between PAK measurements and actual dioptric power for the various measurement conditions in experiments 1,2 , and 3 .

The method of Harris conceals any positive or negative measurement bias. Therefore, the difference in spherical equivalent between PAK measurements of the toric surfaces and the known spherical equivalent of the toric surfaces was also summarised. This preserved the sign of the difference in order to evaluate 
Table 1 Repeated measurements of a toric surface with 43.00 D base curve, $5 \mathrm{D}$ cylinder, axis 90 degrees

\begin{tabular}{|c|c|c|c|c|c|}
\hline \multirow{2}{*}{$\begin{array}{l}\text { Working } \\
\text { distance } \\
(\mathrm{mm})\end{array}$} & \multicolumn{5}{|c|}{$P A K$ corneal measurement } \\
\hline & $\begin{array}{l}\text { Sphere } \\
\text { (D) }\end{array}$ & $\begin{array}{l}\text { Cylinder } \\
\text { (D) }\end{array}$ & $\begin{array}{l}\text { Axis } \\
\text { (deg) }\end{array}$ & $\begin{array}{l}\text { Deviation } \\
\text { (D) }\end{array}$ & $\begin{array}{l}\text { Biast } \\
\text { (D) }\end{array}$ \\
\hline 17 & 43.00 & $5 \cdot 12$ & 91 & $0 \cdot 17$ & +0.06 \\
\hline 18 & $43 \cdot 12$ & $5 \cdot 13$ & 92 & 0.37 & +0.18 \\
\hline 19 & $43 \cdot 12$ & $5 \cdot 13$ & 93 & 0.47 & +0.18 \\
\hline 20 & 43.00 & $5 \cdot 25$ & 93 & 0.45 & +0.12 \\
\hline 21 & 43.00 & $5 \cdot 25$ & 92 & 0.36 & +0.12 \\
\hline 22 & $43 \cdot 12$ & $5 \cdot 13$ & 92 & $0 \cdot 37$ & +0.18 \\
\hline 23 & 43.00 & $5 \cdot 25$ & 92 & 0.36 & +0.12 \\
\hline 24 & 43.00 & $5 \cdot 25$ & 92 & 0.36 & +0.12 \\
\hline 25 & 43.00 & $5 \cdot 25$ & 92 & $0 \cdot 36$ & +0.12 \\
\hline 26 & 43.00 & $5 \cdot 25$ & 92 & 0.36 & +0.12 \\
\hline 27 & 43.00 & $5 \cdot 25$ & 90 & 0.25 & +0.12 \\
\hline 28 & 43.00 & $5 \cdot 12$ & 91 & $0 \cdot 17$ & +0.06 \\
\hline 29 & 43.00 & $5 \cdot 25$ & 92 & 0.36 & +0.12 \\
\hline 30 & $42 \cdot 87$ & $5 \cdot 38$ & 90 & $0 \cdot 28$ & +0.06 \\
\hline
\end{tabular}

*Calculated according to the method of Harris. ${ }^{16}$ †Difference in spherical equivalent between PAK measurement and known surface spherical equivalent.

the magnitude and direction of systematic bias, if present.

For measurements from child and adult subjects, dioptric distances of the observed five points in the cluster from the mean location of points in the cluster were used to determine reproducibility of measurements (method of Harris ${ }^{16}$ ). A Mann-Whitney rank sum test was used to compare reproducibility data for child versus adult subjects - that is, to determine whether the deviations between a subject's PAK readings and that subject's mean dioptric error were significantly greater for children than for adults, and Spearman correlation was used to evaluate the relation between subject age and average measurement deviation, and the relation between operator practice effects and measurement deviation. In addition, the difference between each subject's five measurements of spherical equivalent from their median measurement of spherical equivalent was plotted to determine if outliers might be easily identified in screening situations.

\section{Results}

\section{MEASUREMENTS OF TORIC REFERENCE SURFACES}

\section{Experiment 1: measurement distance}

The closest distance at which the PAK could obtain a measurement was $17 \mathrm{~mm}$ and the furthest distance was $30 \mathrm{~mm}$. Table 1 shows the recorded PAK measurements and calculated dioptric deviations ${ }^{16}$ from the actual

Table 2 Accuracy of PAK under mounted and hand held conditions

\begin{tabular}{lll}
\hline & \multicolumn{2}{l}{$\begin{array}{l}\text { Median measurement deviation }(D)^{\star} \\
(10 \text { measurements per condition })\end{array}$} \\
\cline { 2 - 3 } $\begin{array}{l}\text { Reference } \\
\text { surface }\end{array}$ & $\begin{array}{l}\text { Mounted } \\
(\bar{X} \text { bias, } D) \dagger\end{array}$ & $\begin{array}{l}\text { Hand held } \\
(\bar{X} \text { bias, } D) \dagger\end{array}$ \\
\hline 1 Dioptre & $0 \cdot 39(+0 \cdot 24)$ & $0 \cdot 44(+0 \cdot 28)$ \\
3 Dioptres & $0 \cdot 12(+0 \cdot 05)$ & $0 \cdot 47(+0 \cdot 13)$ \\
5 Dioptres & $0 \cdot 47(+0 \cdot 17)$ & $0 \cdot 37(+0 \cdot 18)$ \\
7 Dioptres & $0 \cdot 59(+0 \cdot 15)$ & $0 \cdot 48(+0 \cdot 18)$ \\
\hline *Calculated according to the method of Harris. & 16 \\
†Difference between PAK measured spherical equivalent and \\
known surface spherical equivalent were calculated and then \\
averaged.
\end{tabular}

reference surface value as the distance from the toric surface to the face plate was varied from 17 to $30 \mathrm{~mm}$. Distance and deviation from actual reference surface toricity were not significantly correlated $\left(r_{\mathrm{s}}=-0 \cdot 47, \mathrm{n}=14\right)$. The median of these distances $(23 \mathrm{~mm})$ was used as the 'optimum' working distance for frame mounted PAK measurements obtained in experiments 2 and 3.

Table 1 also summarises the amount of measurement bias - that is, difference in spherical equivalent between measured and actual surface value. These data indicate a small but consistent positive bias, or an overestimation of spherical equivalent.

\section{Experiment 2: mounted $\mathrm{v}$ hand held measurements}

Table 2 summarises the median deviations from actual reference surface values for PAK mounted and hand held measurements of the four toric surfaces. A Kruskal-Wallis ANOVA on ranks comparing measurement deviation between the four reference surfaces yielded a significant effect $(\mathrm{H}(3)=18.919, \mathrm{p}<0.0004)$. Post hoc comparisons indicated greater measurement deviation for the $7 \mathrm{D}$ reference surface than the 1,3 , and $5 \mathrm{D}$ surfaces, and smaller measurement deviation for the $3 \mathrm{D}$ surface than the 1 and $5 \mathrm{D}$ surfaces. A MannWhitney rank sum test indicated no significant difference in measurement deviation between hand held and mounted measurements. Table 2 also summarises the average measurement bias for each condition. These data indicate a small but consistent positive bias in measuring spherical equivalent.

\section{Experiment 3: angle of eccentricity}

Table 3 summarises the median deviations from actual reference surface values for measurements taken with the $5 \mathrm{D}$ reference surface rotated at various angles of eccentricity away from the PAK. A Kruskal-Wallis ANOVA on ranks comparing measurement deviation across degrees of measurement eccentricity $(0,5,10,15$, and 20 degrees) yielded a significant effect $(\mathrm{H}(4)=12 \cdot 515$, $\mathrm{p}<0 \cdot 02)$. Post hoc comparisons indicated that measurements at 5 and 10 degrees of eccentricity yielded less measurement deviation than measurements at 0,15 , and 20

Table 3 Accuracy of PAK for measuring toric surfaces: effect of eccentricity and axis orientation on measurements of the $5 \mathrm{D}$ reference surface

\begin{tabular}{lll}
\hline & \multicolumn{2}{l}{$\begin{array}{l}\text { Median measurement deviation }(D) \star \\
(2 \text { measurements per condition })\end{array}$} \\
\cline { 2 - 3 } $\begin{array}{ll}\text { Angle of } \\
\text { eccentricity (deg) }\end{array}$ & $\begin{array}{l}\text { Horizontal axis } \\
(\bar{X} \text { bias, } D) \dagger\end{array}$ & $\begin{array}{l}\text { Oblique axis } \\
(\bar{X} \text { bias, } D) \dagger\end{array}$ \\
\hline 0 & $0.41(+0 \cdot 09)$ & $0 \cdot 52(+0 \cdot 18)$ \\
5 & $0.43(+0 \cdot 25)$ & $0 \cdot 28(+0.12)$ \\
10 & $0.15(+0.06)$ & $0 \cdot 37(+0.18)$ \\
15 & $0.46(+0 \cdot 24)$ & $0 \cdot 56(+0.06)$ \\
20 & $0.43(+0 \cdot 12)$ & $0.56(-0.03$
\end{tabular}

${ }^{\star}$ Calculated according to the method of Harris. ${ }^{16}$ †Difference between PAK measured spherical equivalent and known surface spherical equivalent were calculated and then averaged. 


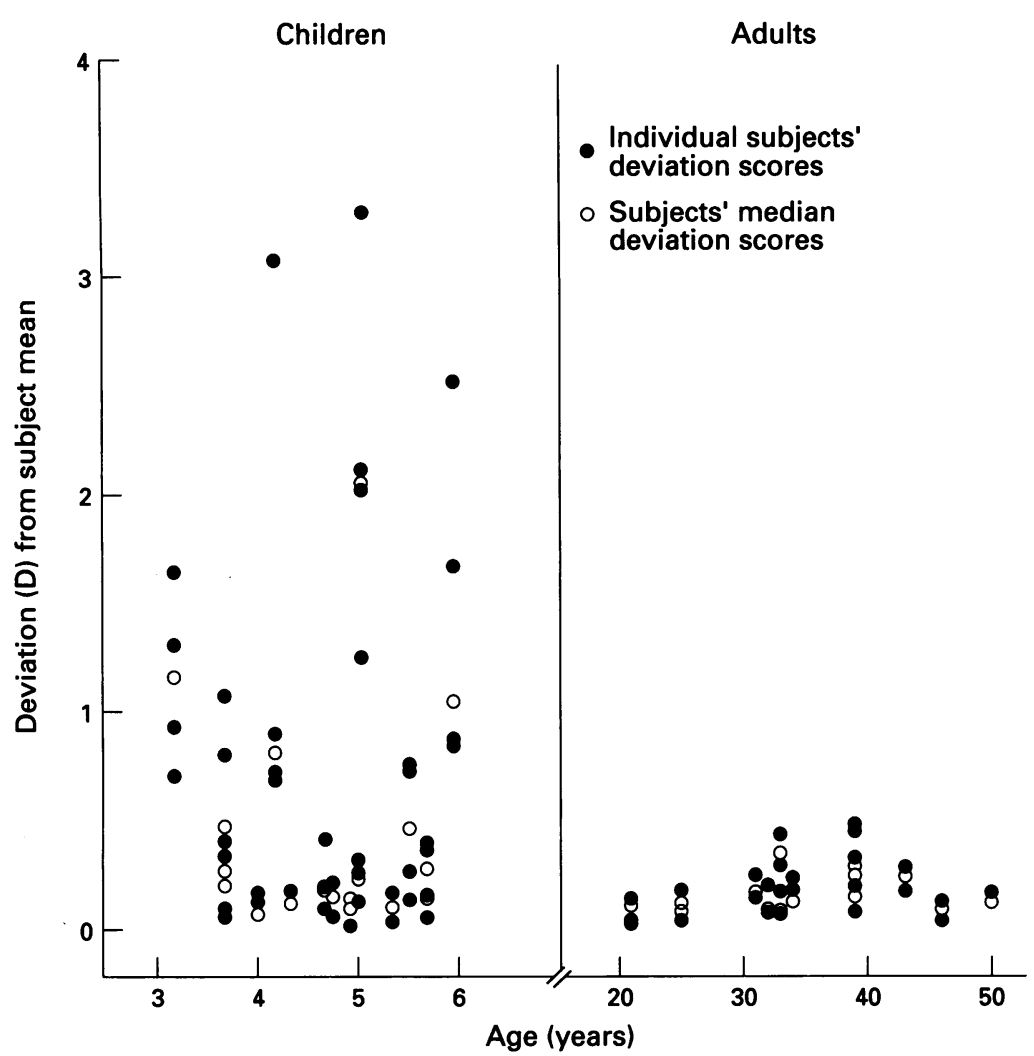

Figure 2 Individual subjects' five deviation scores plotted by age of subject. Filled circles represent the difference in calculated dioptres ${ }^{16}$ between a PAK measurement of a subject's astigmatism and the average dioptric value of the five PAK measurements for that subject. Open symbols represent each subject's median deviation score.

degrees of eccentricity. A Mann-Whitney rank sum test indicated no significant difference in measurement deviation between measurements of horizontally and obliquely oriented axes of toricity.

Table 3 also summarises the average measurement bias for each condition. These data are consistent with the bias data obtained in experiments 1 and 2 - that is, the PAK consistently provides a small amount of overestimation of spherical equivalent.

\section{MEASUREMENTS FROM INFANT, PRESCHOOL, AND ADULT SUBJECTS}

\section{Success in obtaining measurements}

The examiner was successful in obtaining two readings from each eye of one newborn, but was unable to obtain readings from the remaining four newborns tested. Difficulties encountered were related to characteristics of newborn infants, not to examiner difficulties in using the PAK. Specifically, newborns made frequent eye movements and did not fixate the PAK stimulus display. Also, newborns frequently did not spontaneously open their eyes wide enough to allow the entire PAK stimulus display to be imaged on the cornea.

The examiner was successful in obtaining five readings from one eye of 18 children. One child refused to allow the examiner to attempt any measurements. Some children initially had difficulty maintaining fixation. However, with coaching from the examiner and/or parent, ability to achieve fixation improved, and measurements were obtained.
The examiner was successful in obtaining five readings from one eye of all 14 adult subjects.

\section{Variability of estimates}

For the one newborn on whom readings were obtained, the deviation from the mean of the two readings of the right eye was $2.77 \mathrm{D}$ and the deviation from the mean of the two readings of the left eye was $0.83 \mathrm{D}$.

For preschool and adult subjects, the dioptric deviations of each of a subject's five measurements of astigmatism from that subject's mean astigmatism measurement are shown in Figure 2 (filled circles). Also shown in Figure 2 is the median of each subject's five measurement deviations (open circles). A Mann-Whitney rank sum test indicated no significant difference between the median deviation values for children and adults. The median of the 18 median deviation values for child subjects was $0.21 \mathrm{D}$. The median of the 14 median deviation values for adult subjects was $0 \cdot 13 \mathrm{D}$.

In Figure 2, the deviation scores of each of the preschool subjects are plotted by age. There was no significant correlation between age and median deviation scores $\left(r_{\mathrm{s}}=-0.03\right.$, $\mathrm{n}=18$ ) and there was no significant correlation between order in which subjects were tested and median deviation values for either the child or adult groups $\left(r_{\mathrm{s}}=-0 \cdot 29, \mathrm{n}=18\right.$ and $r_{\mathrm{s}}=+0 \cdot 22, \mathrm{n}=14$ for child and adult groups, respectively).

Figure 3 plots the difference between each subject's five measurements of spherical equivalent and that subject's median measurement of spherical equivalent. For children, $85 \%$ of measurements were within $0.3 \mathrm{D}$ from their median, and for adults, $98 \%$ of measurements were within $0.3 \mathrm{D}$ from their median (median measurements not included in calculation of percentages). These data indicate that, although there are more outliers in the child data, both child and adult measurements cluster close to the median measurement.

\section{Discussion}

The PAK is a hand held instrument designed to provide a rapid estimate of corneal astigmatism. Although there have been no published reports in which the PAK was used with young children, the portability and ease of use of the PAK suggest that it might be a useful tool for screening young children for astigmatism. The results of the present study provide data concerning the accuracy of the PAK under a variety of suboptimal conditions likely to occur during screening, as well as data on the usefulness and reliability of the instrument with newborn infants and preschool children.

In the first part of the study, the PAK was used to measure the curvature of $1,3,5$, and 7 D toric reference surfaces. The results of experiment 1 demonstrated little variation in accuracy across the range of distances (from 17 to $30 \mathrm{~mm}$ ) at which the PAK provides a reading (Table 1 ). This is an advantage when screening young children, who may have 


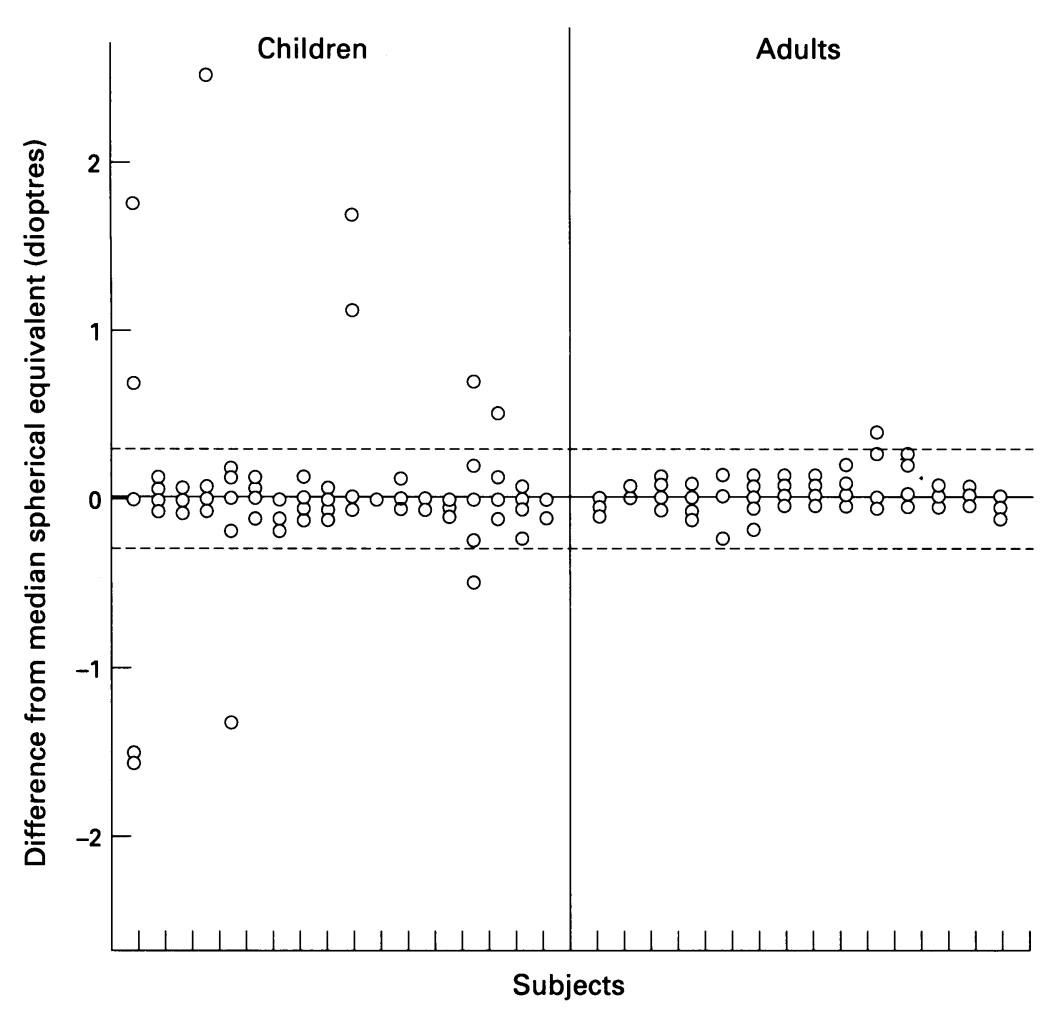

Figure 3 Difference in dioptres between each subject's median measurement of spherical equivalent and each of their five measurements of spherical equivalent. The broken lines represent $+1-0.3 \mathrm{D}$ from the median. difficulty maintaining a constant head position.

The results of experiment 2 yielded a small but significant difference in the accuracy of the PAK across surface curvatures between 1 and $7 \mathrm{D}$ (Table 2). However, accuracy did not appear to be systematically related to increasing amounts of toricity. In addition, we observed no difference in the accuracy of measurements under mounted versus hand held measurements, suggesting little effect of operator misalignment error on accuracy of measurements.

In experiment 3, we observed that, although measurements were significantly less accurate at some angles of eccentricity, accuracy was not systematically related to the magnitude of the angle of eccentricity. This characteristic would be advantageous when screening children, who may not consistently look directly at the fixation target. However, off centre measurements of a regular toric surface may not adequately model off axis measures of a human cornea.

In experiment 3, no difference in accuracy of the PAK was observed for measurements obtained with the axis of astigmatism oriented horizontally versus obliquely with respect to the PAK. This result suggests that the PAK is successful in providing accurate measurements of obliquely oriented toric surfaces, despite the fact that the array of lights used by the PAK to create Purkinje images from the cornea or from the toric surface are oriented along the vertical and horizontal meridia.

Examination of measurement bias in experiments 1,2 , and 3 indicate that the PAK provides a small but consistent overestimate of spherical equivalent. The fact that these overestimates are small indicates that bias would not preclude the use of the PAK as a screening tool. The average bias over all measurements obtained in experiments 1,2 , and 3 was $+0 \cdot 16$ $\mathrm{D}$ (range $=-0.06$ to $+0.37,114$ measurements).

In summary, measurement of toric reference surfaces suggest that the PAK is an accurate and versatile instrument that could be effective in measuring corneal astigmatism in less than optimally cooperative populations.

The goal of the second part of the present study was to study the feasibility and reliability of using the PAK with newborn infants and preschool age children. The results indicated that the PAK was not effective in measuring corneal astigmatism in newborns. Measurements were obtained in only one of the five newborns tested, and in the one newborn on whom measurements were obtained, there was a difference of nearly $3 \mathrm{D}$ between the two measurements obtained on the right eye and a difference of nearly $1 \mathrm{D}$ between the two measurements obtained on the left eye. The primary difficulties in obtaining measurements from newborns were (a) keeping the infant's eye open and (b) attracting fixation in one direction long enough for the PAK to obtain the five consecutive, consistent readings that are required for the instrument to provide a measurement of astigmatism. Modification of the PAK through the addition of a recording system that would permit selective averaging of readings taken only on frames in which the eye was directed at the instrument might overcome the current limitations of the instrument for screening of newborn infants.

In contrast with our unsuccessful results with newborn infants, we were able to achieve our goal of recording five measurements from one eye of all but one of the 19 preschool subjects. For these 18 subjects, the median dioptric deviation between the five measurements and the subject's average measurement of corneal astigmatism ranged from 0.08 to $2.06 \mathrm{D}$ (median of the 18 medians $=0.21 \mathrm{D}$ ). The range for adults' median deviations was 0.09 to $0.35 \mathrm{D}$ (median of the 14 medians $=0.13 \mathrm{D}$ ). Within the group of preschool children, there was no evidence the measurements were less variable for older children and no evidence that variability of measurements decreased as the operator gained more experience in working with children in this age group.

Examination of Figure 3 indicates that both child and adult measurements tend to cluster close to the median measurement. This figure also suggests that outliers can be clearly identified and eliminated if repeated measurements are obtained when screening for corneal astigmatism in young children.

A review of the literature indicated that three techniques other than the PAK have been used as an alternative to traditional keratometry for assessment of corneal astigmatism in children. Nakada et al compared measurements of corneal curvature obtained with the Canon automated keratometer to those obtained with a Bausch \& Lomb conventional keratometer in a study of 140 patients between 3 and 72 years 
of age. ${ }^{17}$ The correlation between measurements obtained with the two techniques was high and, although no data on reproducibility of measurements were provided, the authors concluded that the high correlation between measurements obtained with the two techniques and the ease of use of the automated keratometer make it especially appropriate for assessment of children.

Howland and Sayles used a photokeratometer that they constructed, to assess corneal astigmatism in 103 children between birth and 5 years of age, including 44 infants less than 1 year of age. ${ }^{5}$ Measurements were successfully obtained on all but three subjects. Reproducibility of measurements, assessed by calculating the average difference between two measurements of astigmatism in 19 children, was 0.24 (SD 0.18) D. To compare our results with those of Howland and Sayles, we calculated the difference between the first and second measurements of astigmatism in each subject, and computed the average of these differences. For children, the average difference was $0.38(0.37) \mathrm{D}$, and for adults, the average difference was $0.20(0.14) \mathrm{D}$. Thus, Howland and Sayles reported better reproducibility of measurements in children for the photokeratometer than we report for the PAK. However, a fundamental difference between the photokeratometer and the PAK is that the photokeratometer produces a series of photographs from which the photographs with the best eye alignment can be selected for analysis. Such selection of measurements is not possible with the PAK.

Zadnik et al used photokeratoscopy (KERA 9-ring CorneaScope) to measure corneal curvature in 6 to 12 -year-old children. ${ }^{18}$ In a later study, they reported data on the reproducibility of photokeratoscope measurements in adult subjects, based on calculations of the difference between two measurements of curvature in the vertical meridian of 40 subjects. ${ }^{19}$ For comparison, we calculated the difference between the first two measurements of curvature of the most vertical meridian (K1) in each child and each adult subject in the present study. The results, shown in Table 4, indicate that the reproducibility of PAK measurements in children in the present study was comparable with the reproducibility of photokeratoscopy measurements of adults in the study of Zadnik et al.

In summary, the PAK is more portable than the automated keratometer tested by Nakada et $a l,{ }^{17}$ has higher reproducibility in adult subjects than the photokeratoscope tested by Zadnik et $a l,{ }^{19}$ and has the advantage of being commercially available, in contrast with the photokeratometer developed by Howland and

Table 4 Reproducibility of measurements (difference (D) between two measurements of corneal curvature in the vertical meridian) with photokeratoscopy ${ }^{19}$ and with the $P A K$

\begin{tabular}{llll}
\hline Instrument (subjects) & $\begin{array}{l}\bar{X} \text { Measurement } \\
\text { difference }(D)\end{array}$ & $\begin{array}{l}\text { Standard } \\
\text { deviation }\end{array}$ & $\begin{array}{l}\text { 95\% Confidence } \\
\text { interval }\end{array}$ \\
\hline Photokeratoscope (adults) & -0.24 & 1.02 & -2.24 to 1.76 \\
PAK (preschool children) & -0.35 & 1.06 & -2.43 to 1.73 \\
PAK (adults) & 0.0007 & 0.18 & -0.35 to 0.35 \\
\hline
\end{tabular}

Sayles. ${ }^{5}$ However, reproducibility of measurements produced by the PAK is not as good as that based on selected photographs from the Howland and Sayles photokeratometer, and, unlike the photokeratometer, it has not been demonstrated that the PAK can be used successfully with infants.

\section{Conclusion}

The results of the present study indicate that, firstly, the PAK provides an accurate estimate of the asphericity of a surface, even when measurements are taken under less than optimal conditions. Secondly, the results show that the PAK can be used successfully in children between 3 and 5 years of age, and indicate that the variability in the measurements obtained is low enough that the instrument could be used successfully as a screening tool for corneal astigmatism in children 3 to 5 years of age. The variability in estimates of corneal power in the preschool age group is large enough, however, to indicate that other methods should be used to determine the precise astigmatic correction that should be prescribed for a young child.

Some populations are known to be at risk for astigmatism. For example, studies have demonstrated that certain groups of native Americans ${ }^{20-29}$ have a high prevalence of high astigmatic error, which is primarily corneal in nature. ${ }^{23} 27-29$ Use of a screening instrument such as the PAK would permit early detection of astigmatism in such populations at an age at which optical correction could be implemented to prevent the development of meridional amblyopia.

The authors thank Loretta Nelson and Alcon Systems Inc, for the use of the Alcon portable auto-keratometer and Chris Arslanian, $\mathrm{PhD}, \mathrm{RN}$, for assistance in recruiting newborn subjects.

This research was presented in part at the Association for Research in Vision and Ophthalmology Annual Meeting, Ft Research in Vision and Ophthalmology Annual Meeting, Ft Lauderdale, FL, 18 May 1995. Supported by NIH grant EY05804 (VD) and by a grant from the Whitaker Foundation
JM). The authors have no proprietary interest in the present work.

1 Howland HC, Atkinson J, Braddick O, French J. Infant astigmatism measured by photorefraction. Science 1978; 202: 331-2

2 Mohindra I, Held R, Gwiazda J, Brill S. Astigmatism in infants. Science 1978; 202: 329-30.

3 Fulton AB, Dobson V, Salem D, Mar C, Petersen RA, Hansen RM. Cycloplegic refractions in infants and young children. Am f Ophthalmol 1980; 90: 239-47.

4 Howland HC, Sayles N. Photorefractive measurements of astigmatism in infants and young children. Invest Ophthalmol Vis Sci 1984; 25: 93-102.

5 Howland HC, Sayles N. Photokeratometric and photorefractive measurements of astigmatism in infants and young children. Vision Res 1985; 25: 73-81.

6 Gwiazda J, Scheiman M, Mohindra I, Held R. Astigmatism in children: changes in axis and amount from birth to six in children: changes in axis and amount from birth

7 Atkinson J, Braddick O, French J. Infant astigmatism: its disappearance with age. Vision Res 1980; 20: 891-3.

8 Gwiazda J, Bauer J, Thorn F, Held R. Meridional amblyopia does result from astigmatism in early childhood. Clin Vis Sci 1986; 1: 145-52.

9 Freeman RD, Mitchell DE, Millodot M. A neural effect of partial visual deprivation in humans. Science 1972; 175: 1384-6.

10 Mitchell DE, Freeman RD, Millodot M, Haegerstrom G. Meridional amblyopia: evidence for modification of the human visual system by early visual experience. Vision Res 1973; 13: 535-58.

11 Cobb SR, MacDonald CF. Resolution acuity in astigmats: evidence for a critical period in the human visual system. Br f Physiol Opt 1978; 32: 38-49.

12 Mohindra I, Jacobson SG, Held R. Binocular visual form deprivation in human infants. Doc Ophthalmol 1983; 55: $237-49$. 
13 Palmer ML, Reid TK. Reproducibility and accuracy of normal corneal power measurements with a portable automated keratometer versus a videokeratoscope. Invest Ophthalmol Vis Sci 1994; 35 (suppl): 2194.

14 Alcon auto-keratometer operator's manual. St Louis: Alcon Systems Inc, 1993.

15 Greivenkamp JE, Mellinger MD, Snyder RW, Miller JM. Measurement of toric reference surfaces with the computed anatomy videokeratoscope (TMS-1). In: Noninvasive assessment of the visual system technical digest, 1993. Washington, DC: Optical Society of America, 1993; 3: 54-7.

16 Harris WF. Algebra of sphero-cylinders and refractive errors, and their means, variance and standard deviation. Am f Optom Phys Optics 1988; 65: 794-802.

17 Nakada S, Minoru T, Nakajima A. Comparison of automated and conventional keratometers. Am $\mathcal{f}$ Ophthalmol 1984; 97: 776-8.

18 Zadnik K, Satariano WA, Mutti DO, Sholtz RI, Adams AJ The effect of parental history of myopia on children's eye
size. $¥ A M A$ 1994; $271: 1323-7$.

19 Zadnik K, Mutti DO, Adams AJ. The repeatability of measurements of the ocular components. Invest Ophthalmol Vis Sci 1992; 33: 2325-33.
20 Levy WJ, Wall FJ. A study of the refractive state of a group of American Pueblo Indians. Rocky Mountain Medical fournal 1969: 40-2.

21 Abraham JE, Volovick JB. Preliminary Navajo optometric study. F Am Optom Assoc 1972; 43: 1257-60.

22 Boniuk V. Refractive problems in native peoples (the Sioux Lookout Project). Can f Ophthalmol 1973; 8: 229-33.

23 Kershner RM, Brick DC. Prevalence of high corneal astigmatism in Papago school children. Invest Ophthalmol Vis Sci 1984; 25 (suppl): 217.

24 Wick B, Crane S. A vision profile of American Indian children. $\mathcal{f}$ Optom Physiol Opt 1976; 53: 34-40.

25 Hamilton JE. Vision anomalies of Indian school children: the Lame Deer study. 7 Am Optom Assoc 1976; 47: 479-87.

26 Mohindra I, Nagaraj S. Astigmatism in Zuni and Navajo Indians. Am f Optom Physiol Opt 1977; 54: 121-4.

27 Lyle WM, Grosvenor T, Dean KC. Corneal astigmatism in Amerind children. Am $¥$ Optom Arch Am Acad Optom 1972; 49: 517-24.

28 Garber JM. High corneal astigmatism in Navajo school children and its effect on classroom performance. $\mathcal{F} \mathrm{Am}$ Optom Assoc 1981; 52: 583-6.

29 Heard T, Reber N, Levi D, Allen D. The refractive status of Zuni Indian children. Am $\mathcal{f}$ Optom Physiol Opt 1976; 53: 120-3. 